\title{
Pulmonary thromboembolism with multiple right heart mural thrombus in a patient with COVID-19
}

\author{
Houman Dehghan ${ }^{1} \cdot$ Azam Soleimani $^{1,2}$ (B)
}

Received: 24 August 2020 / Revised: 12 October 2020 / Accepted: 14 October 2020 / Published online: 26 October 2020

(c) Japanese Society of Echocardiography 2020

Hypercoagulation state during COVID-19 disease has been confirmed recently. Herein we present a 40 -year-old hypertensive otherwise healthy male who was admitted with complaints of myalgia, low grade fever, dry cough, leg swelling, and exacerbating dyspnea in the last two weeks with history of recent recovery from COVID-19 in his wife. At presentation, he was afebrile with stable vital signs (blood pressure: $114 / 81 \mathrm{mmHg}$, respiratory rate: $16 /$ minute, heart rate: $75 \mathrm{bpm})$ despite hypoxemia $\left(\mathrm{SPaO}_{2}\right.$ of $74 \%$ on room air, $94 \%$ on nasal O2 therapy). Coarse crackles in the lung fields, symmetric pitting leg edema and elevated jugular venous pressure were noticed in clinical exam with low voltage QRS, extreme right axis deviation and negative $\mathrm{T}$ waves in the precordial leads in electrocardiogram (Fig. 1a). Chest CT scan demonstrated pericardial and pleural effusions, filling defects in right atrium (RA) and right ventricle (RV) cavities, peripheral based ground glass opacities (compatible with COVID involvement), wedge-shaped pulmonary infarct and pulmonary arterial branch thrombosis (Fig. 1b, supplementary video1). No deep vein thrombosis was found. Transthoracic and transesophageal echocardiography showed preserved left ventricular systolic function, flattened interventricular septum, moderate right atrium (RA) and right ventricular (RV) enlargement and dysfunction, plethoric inferior vena cava with two large mural thrombi

Electronic supplementary material The online version of this article (https://doi.org/10.1007/s12574-020-00500-x) contains supplementary material, which is available to authorized users.

Azam Soleimani

asoleimanii@gmail.com

1 Fellowship of Echocardiography, Echocardiography department, Chamran Cardiovascular Medical and Research Center, Isfahan University of Medical Sciences, Isfahan, Iran

2 Fellowship of Echocardiography, Cardiac Rehabilitation Research Center, Isfahan Cardiovascular Research Institute, Isfahan University of Medical Sciences, Isfahan, Iran
$(4 \mathrm{~cm} \times 2.5 \mathrm{~cm})$ in RA appendage and RV apex (Fig. 1c, d, supplementary videos 2,3 ).

In laboratory data, positive oropharyngeal swab for severe acute respiratory syndrome corona virus $2\left(\mathrm{SARS}-\mathrm{COV}_{2}\right)$, lymphopenia (782 per $\mu \mathrm{L}$ ) without leukocytosis ( 4150 per $\mu \mathrm{L})$, elevated d-dimer $(4510 \mathrm{ng} / \mathrm{ml}$, normal $<250)$, cardiac troponin I $(115 \mathrm{ng} / \mathrm{L}$, normal $<50)$ and $\mathrm{C}$-reactive protein $(18.9 \mathrm{mg} / \mathrm{L}$, normal $<5)$ were observed.

Under full intravenous anticoagulation and oral vitamin $\mathrm{K}$ antagonist the thrombus size was reduced significantly and RV function was improved.

COVID-19 disease, has been proposed to be associated with cardiovascular manifestations including acute coronary syndrome, arrhythmia, myocarditis and thromboembolism [1]. Hypercoagulation state during COVID-19 is attributed to systemic inflammation, immobility and hypoxemia [2]. The theory of endothelial lung injury along with micro thrombus formation in pulmonary vascular bed, leads to the general agreement for anticoagulant therapy in critically ill hospitalized patients with COVID-19 as a standard prophylactic approach [3].

Although there are reports of successful thrombolytic therapy in unstableCOVID-19 patients with right heart thrombus, the stable subacute presentation of the patient, leads us to follow conservative approach $[4,5]$.

This patient presented with pulmonary thromboembolism accompanied by multiple large right heart thrombus represents a unique serious form of cardiopulmonary injury during COVID-19 disease. 


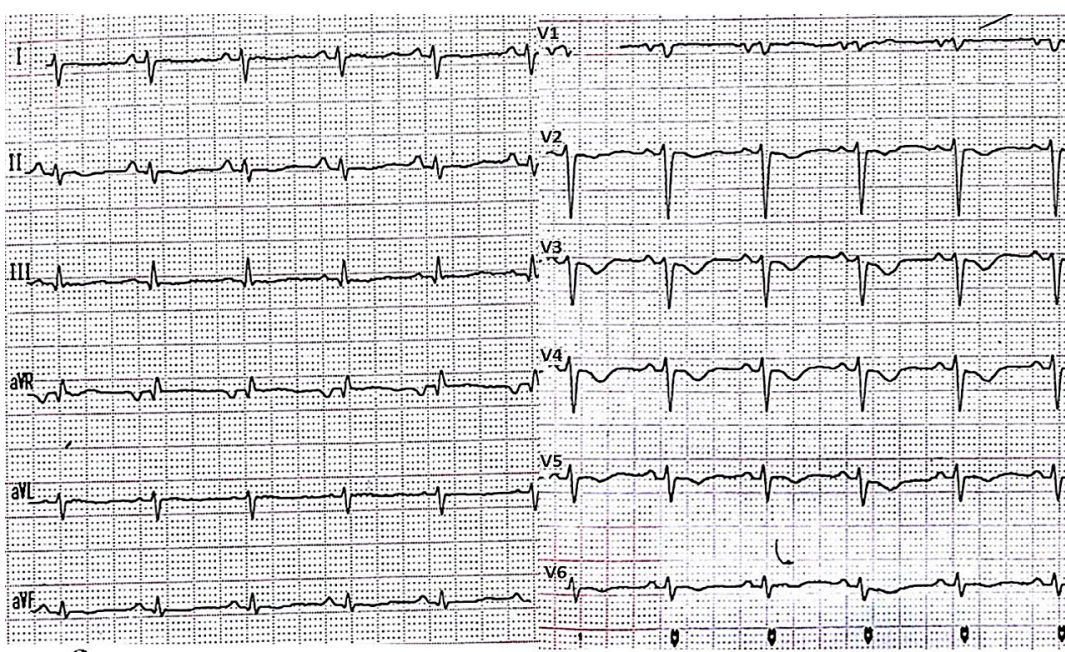

a

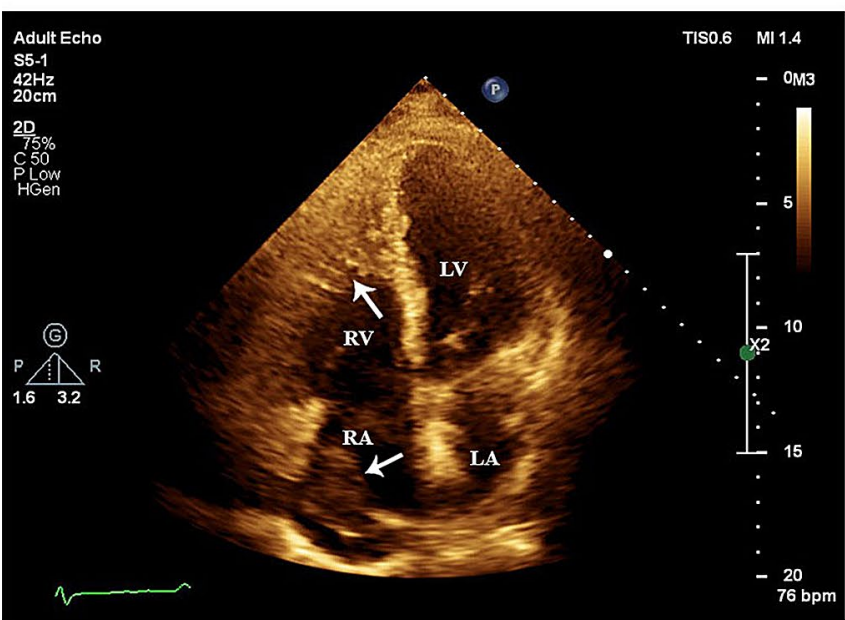

c

Fig. 1 a Surface electrocardiogram shows normal sinus rhythm, low voltage $\mathrm{QRS}$, extreme right axis deviation and negative $\mathrm{T}$ waves in the precordial leads. b Mid thoracic slice of chest CT scan demonstrated moderate pericardial and bilateral pleural effusions, peripheral based patchy ground glass opacities and wedge-shaped pul-

\section{Compliance with ethical standards}

Conflict of interest Houman Dehghan and Azam Soleimani declare that they have no conflict of interest.

Human rights statements and informed consent All procedures followed were in accordance with the ethical standards of the responsible committee on human experimentation (institutional and national) and with the Helsinki Declaration of 1964 and later versions. Informed consent was obtained from the patient for being included in the study.

\section{Reference}

1. Hendren NS, Drazner MH, Bozkurt B, et al. Description and proposed management of the acute COVID-19 cardiovascular syndrome. Circulation. 2020;141(23):1903-14.

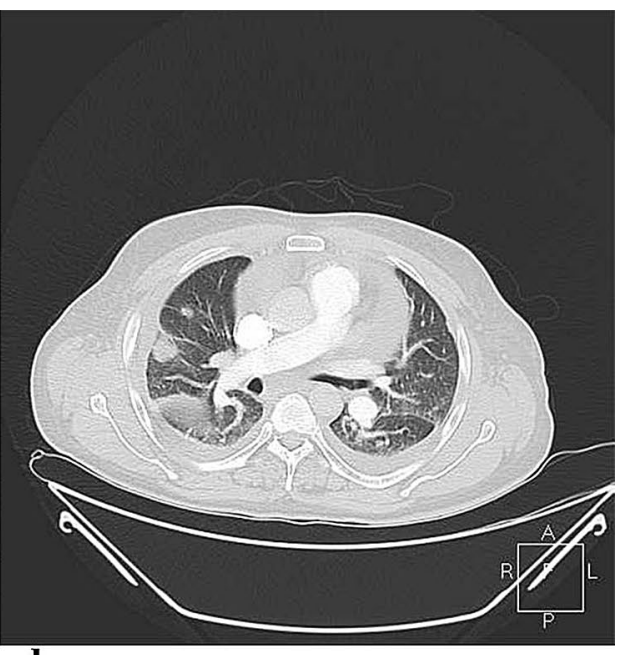

b

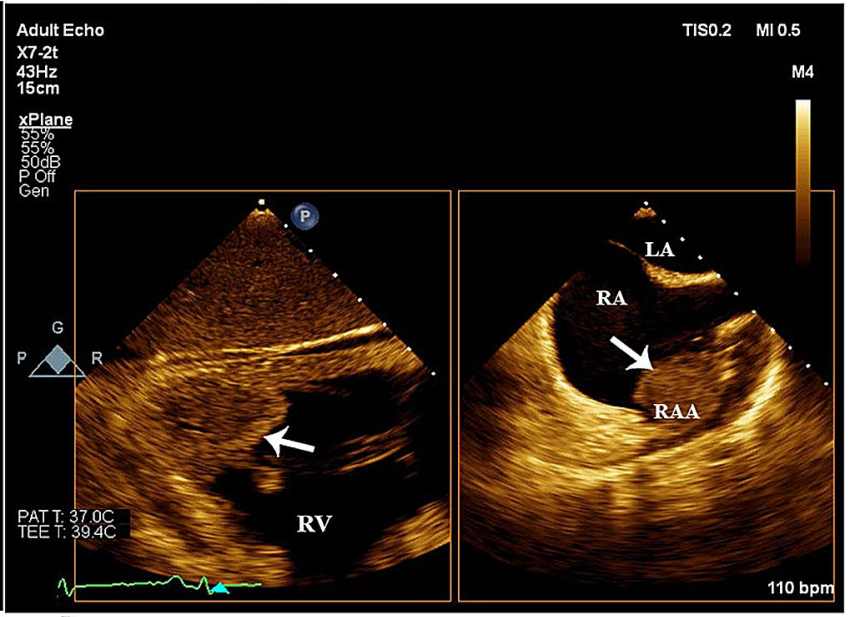

d

monary infarct in the right lower lobe. $\mathbf{c}$ and $\mathbf{d}$ Transthoracic and transesophageal echocardiography (trans gastric and mid esophageal bicaval views) shows two large mural thrombus (average size of $4 \mathrm{~cm} \times 2.5 \mathrm{~cm}$ ) in RA appendage (RAA) and RV apex along with small circumferential pericardial effusion

2. The European society for cardiology. ESC guidance for the diagnosis and management of CV disease during the COVID-19 pandemic. https://www.escardio.org/Education/COVID-19-and-Cardi ology/ESC-COVID-19-Guidance. (Last update: 10 June 2020)

3. Moores LK, Tritschler T, Brosnahan S, et al. Prevention, diagnosis, and treatment of vte in patients with coronavirus disease 2019: CHEST guideline and expert panel report. Chest. 2020;158(3):1143-63.

4. Sethi SS, Zilinyi R, Green P, et al. Right ventricular clot in transit in COVID-19 Implications for the pulmonary embolism response team. JACC Case Rep. 2020;2(9):1391-6.

5. Janus SE, Hajjari J, Cunningham MJ, Hoit BD. COVID19: a case report of thrombus in transit. Eur Heart J Case Rep. 2020;4(FI1):1-4.

Publisher's Note Springer Nature remains neutral with regard to jurisdictional claims in published maps and institutional affiliations. 